\title{
Spring-bead Animation of Viscoelastic Materials
}

\author{
Nobuhiko Tamura \\ Norimichi Tsumura \\ Toshiya Nakaguchi \\ Yoichi Miyake
}

Chiba University, JAPAN

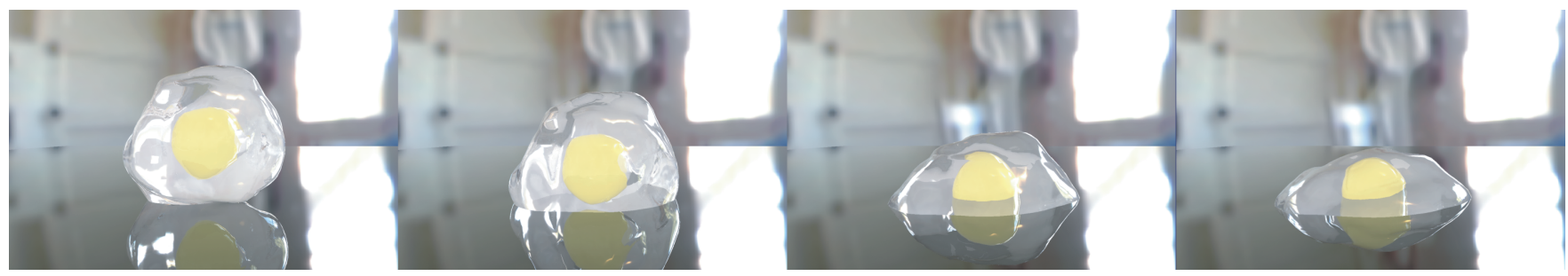

Figure 1: Egg dropped onto the floor. The yolk (20 connections per particle) is covered by the egg white (4 connections per particle).

\section{Overview}

Polymeric materials such as rubber, fat, oil and egg are known to have viscoelastic characteristics. This sketch presents a method to simulate viscoelastic material using a spring-mass system. A large number of particles $(20,000)$ are used to create a randomly connected mesh that mimics the structure of the polymeric material. The resulting spring-mass system can be simulated efficiently on a graphics processing unit (GPU).

\section{Spring-bead model of polymeric material}

Polymeric material consists of polymer chains in which a number of polymer units are connected together and act like a chain. The spring-bead model [Rouse 1953] is one of the simplest models of a polymer chain. Rouse's model divides a single polymer chain into subsections. Each subsection is replaced by beads (elements which represent friction, mass and volume). The neighboring beads are connected by springs (elements which represent rubber-like behavior for small elongations). Rouse's model was originally used to calculate the entropy of the polymer system using statistical mechanics, i.e., it was not used for calculating the macroscopic shape of polymeric material. Actually, Rouse's model can be considered to be a simple spring-mass chain. However, we call our method the spring-bead method because our method is inspired by the work of Rouse. Obviously, a one-dimensional chain does not support a 3D structure. A real polymeric material obtains its 3D structure by twines of polymer chains. Therefore, we modified Rouse's model by adding cross chains. Fig. 2 illustrates our mesh structure. The $\alpha$-spring comes from Rouse's original model and the $\beta$-spring represents the twine. This concept is considered to be analogous to the vulcanization of rubber, whereby the raw sap fluid from the rubber tree is converted to solid rubber by the connection of polymer chains with sulfur. This process increases the elasticity of the fluid, causing the rubber to act as a solid material.

The creation of a random mesh is as follows. First, a polygon is filled with a single chain-like molding. The connection angle of the neighboring spring is random. Second, each particle is connected to the other particles randomly by a $\beta$-spring. The bunny in Fig. 3 contains 20,000 particles and each particle has 20 connections to the other particles ( $2 \alpha$-springs and $18 \beta$-springs). The elasticity can be changed by changing the number of connections (Fig.1).

\section{Spring-mass system on a GPU}

Euler explicit integration is used to simulate the spring-bead model. We used a GPU (GeForce FX6800) for the computation and the computation method of huge particle systems described in [Lutz 2004]. In this method, position and velocity are stored as a texture. The texture is updated by a pixel shader, which implements a simple Euler explicit equation. However, we need to implement the two interacting forces between particles: the spring force and the pressure force. The lookup texture is used to relate a particle ID (texture coordinate) and its connecting particle IDs for the calculation of the spring force. The pressure force is used as a substitute for the collision force. We assumed the pressure force to be the gradient of the 3D histogram of a particle's position. The 3D histogram is calculated using displacement mapping, which is one of the attractive features of Shader Model 3.0; vertices which store the position $(0,0,0)$ and the particle ID are created at initialization. When the vertices are passed to the Vertex Shader program, the position of each particle is fetched from the position texture. The vertices are displaced and rendered to the $3 \mathrm{D}$ texture. Alpha blending is set to create a $3 \mathrm{D}$ histogram of the particles. The $3 \mathrm{D}$ texture is implemented by slicing the volume into $2 \mathrm{D}$ sections.

The animation employs two separate visualization methods for offline and interactive rendering. In offline rendering, the $3 \mathrm{D}$ volume is filled with a scalar field generated by the particles. An iso-surface is created by the marching cubes method. Fig. 1 shows the rendering result. Slice-based volume rendering is employed for interactive rendering. Because the computational cost for interactive surface extraction is not negligible, the 3D histogram of the particles for the pressure calculation is re-used for visualization. Slices of the $3 \mathrm{D}$ histogram are aligned along the axis. Alpha blending is set to combine the slice images, and shading is executed by treating the gradient of the 3D histogram as a normal vector. Fig. 3 shows a bunny interacting with a solid sphere. This interactive animation was done at $30 \mathrm{fps}$. The bunny is melted by cutting the springs. With the improvement of graphics hardware, a more refined rendering will be possible with high-resolution $3 \mathrm{D}$ textures.

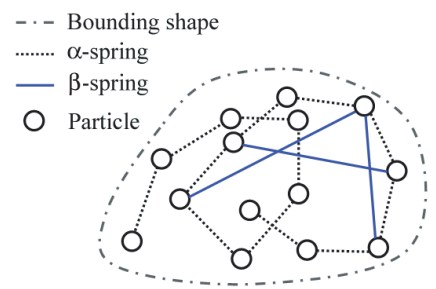

Figure 2: Modified spring-bead model of Rouse

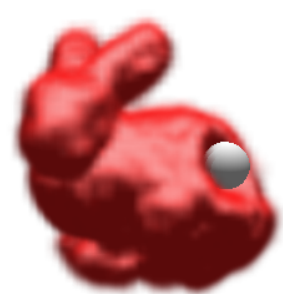

Figure 3: Real-time animation of the viscoelastic bunny model

\section{References}

Rouse, P. E., 1953, A Theory of the Linear Viscoelastic Properties of Dilute Solutions of Coiling Polymers, In J. Chem. Phys, 21, 1272.

Lutz, L., 2004, Building a Million Particle S ystem, In Proceedings of GDC2004. 